\title{
Anemia por deficiencia de hierro en mujeres mexicanas en edad reproductiva. Historia de un problema no resuelto
}

\author{
Esther Casanueva, N utr, D ra en Epidemiología, (1) Luz María de Regil, N utr, MC, (1) \\ María Fernanda Flores-C ampuzano,Act. ${ }^{(1)}$
}

\section{Casanueva E, De Regil LM, Flores-Campuzano MF. Anemia por deficiencia de hierro en mujeres mexicanas en edad reproductiva. Historia de un problema no resuelto. Salud Publica Mex 2006;48:166-175.}

\begin{abstract}
Resumen
Objetivo. D escribir la prevalencia informada de anemia por deficiencia de hierro en mujeres mexicanas en edad reproductiva en los últimos 66 años y analizar la eficacia de las intervenciones informadas para su prevención y manejo. Material y métodos. Revisión de estudios epidemiológicos obser vacionales, clínicos y programas gubernamentales de intervención, publicados entre 1939 y 2005 con información original. Se consultaron las bases de datos de la Biblioteca $\mathrm{N}$ acional de Medicina de Estados Unidos, Artemisa e índices de revistas mexicanas. La calidad de los ensayos clínicos se evaluó con base en la escala Jadad. Las tendencias de la prevalencia de anemia se analizaron con una regresión lineal ponderada por el tamaño muestral. Resultados Se incluyeron 46 trabajos de investigación; nueve correspondieron a ensayos clínicos y cuatro a programas operativos; 17 informaron la prevalencia de anemia en no embarazadas y 23 en gestantes. En el primer grupo, la prevalencia ponderada de anemia ha descendido de 39.6 a $15.5 \%$, en tanto que en las gestantes ha disminuido 10 puntos porcentuales, hasta 25\%. De los estudios clínicos, $55 \%$ se consideraron con un nivel de evidencia adecuado. Conclusiones La anemia en mujeres en edad reproductiva, y particularmente en las embarazadas, aún constituye un problema de salud pública. De seguir con los esquemas actuales, se requerirían cerca de 57 años para erradicar la anemia entre las no gestantes y 121 para las gestantes. Es necesario evaluar las estrategias de intervención y hacer estudios consistentes que permitan tomar las medidas adecuadas para controlarla.
\end{abstract}

Palabras clave: anemia; mujeres; embar azo; reproductiva; revisión sistemática; México

\section{Casanueva E, De Regil LM, Flores-Campuzano MF. Iron deficiency anemia among Mexican women on reproductive age. History of an unresolved problem. Salud Publica Mex 2006;48:166-175.}

\begin{abstract}
A bstract
Objective. To describe the prevalence of iron deficiency anemia in the past 66 years among Mexican women on reproductive age, and to analyze the efficacy of interventions implemented for its prevention and control. Material and Methods 0 bservational and clinical epidemiological studies as well as federal intervention programs published between 1939 and 2005 having original data related to anemia were review ed using electronic databases (Medline and Artemisa) and manual searches. Keywords were anemia, iron deficiency, women, and Mexico.The quality of clinical trials was evaluated using the Jadad scale.A nemia prevalence tendency was analyzed using a linear regression weighted according to the sample size. Results Forty-six research studies were included, nine corresponded to clinical trials and four to federal-operative programs. Seventeen papers reported anemia prevalence in non-pregnant women and twenty-three in pregnant women. In the first group, weighted anemia prevalence had decreased from 39.6 to $15.5 \%$, whereas in pregnant women it decreased from 35 to $25 \%$. of the clinical trials, $55 \%$ were considered to be good quality studies. Conclusions A nemia in women on reproductive age, particularly in pregnant women, is still a public health problem in Mexico. If the current conditons continue, it would take 57 years to eradicate anemia in non-pregnant and 121 years in pregnant women. It is necessary to evaluate intervention policies and conduct consistent studies in order to enable appropriate steps to be taken to control anemia.
\end{abstract}

Key words: anemia; women; pregnancy; reproductive; systematic review; Mexico

(1) Instituto $\mathrm{N}$ acional de Perinatología, México.

Fecha de recibido: 19 de julio de 2005 - Fecha de aprobado: 28 de noviembre de 2005 Solicitud de sobretiros: Dra. Esther C asanueva. Instituto N acional de Perinatología Isidro Espinosa de los Reyes. Montes Urales 800, colonia Lomas de Virreyes. Miguel Hidalgo 11000 México, D F, México. Correo electrónico: casanuev@ servidor.unam.mx 
$\Lambda$ pesar de que, por razones metodológicas, ${ }^{1}$ no se han podido establecer los mecanismos mediante los cuales se asocia la anemia con el pobre desempeño reproductivo, es un hecho bien conocido que las mujeres con anemia por deficiencia de hierro tienen productos prematuros o con bajo peso al nacer, con una frecuencia significativamente mayor que las no anémicas. ${ }^{2-4}$ De igual manera, se ha documentado que las mujeres con reservas pregestacionales insuficientes de hierro incrementarán su riesgo de padecer anemia durante el embarazo ${ }^{5,6}$ además de tener menor capacidad para realizar actividad física, ${ }^{7}$ o mayor susceptibilidad a presentar infecciones ${ }^{8}$ Inclusive, en fechas recientes se ha informado que las madres con anemia por deficiencia de hierro tienen una pobre interacción con sus hijos. ${ }^{9}$ En consideración a lo anterior, la prevención de la anemia por deficiencia de hierro debe constituir una de las prioridades de los programas de salud y nutrición de la mujer en edad reproductiva.

De acuerdo con los lineamientos de diversos organismos, la mejor forma de implementar un programa de prevención y tratamiento de anemia por deficiencia de hierro es partiendo de un diagnóstico adecuado de la situación e identificando a los grupos con mayor riesgo para, después, establecer medidas que den resultado en el corto, mediano y largo plazo. En el caso de la prevención de la anemia por deficiencia de hierro en mujeres en edad reproductiva, se ha recomendado para el corto plazo el establecimiento de programas de suplementación, la promoción del espaciamiento entre embarazos, el control prenatal oportuno y la desparasitación periódica en las comunidades que así lo requieran. Para el mediano plazo se identifica el establecimiento de una política de adición de hierro a los alimentos y la promoción de los programas de sanidad e higiene. Por último, dentro de las tareas con resultados en el largo plazo se recomienda la promoción del consumo de una dieta completa y diversificada. ${ }^{10-12}$

En México no se ha logrado una prevención efectiva de la anemia por deficiencia de hierro en mujeres en edad reproductiva, probablemente porque las acciones antes descritas no se han llevado a cabo de manera sistemática y ordenada. Así entonces, el objetivo de este trabajo es describir la prevalencia informada de anemia por deficiencia de hierro en mujeres mexicanas en edad reproductiva en los últimos 66 años y analizar la eficacia de las intervenciones informadas para su prevención y manejo.

\section{Material y métodos}

La revisión se llevó a cabo de acuerdo con las guías sugeridas para el informe de meta-análisis de estudios epidemiológicos observacionales (MOOSE, por sus siglas en inglés). ${ }^{13}$

En la identificación de los estudios publicados se consultaron las bases de datos de la Biblioteca Nacional de Medicina de Estados Unidos de América (Nacional Library of Medicine- Pub-Med http:/ / www. ncbi.nlm.nih.gov/entrez/query) y los discos compactos de ARTEMISA (Artículos Editados en México sobre Información en Salud, 1993 a 2003). En el caso de los artículos publicados antes de 1980, se consultó la propia bibliografía de los artículos identificados y se revisaron, en forma manual, los índices de las revistas mexicanas que pudieran contener artículos sobre el tema : Revista de Investigación Clínica; Ginecología y Obstetricia de México y Gaceta Médica de México. Las palabras clave utilizadas fueron anemia, hierro, mujeres y México, con su respectiva traducción para el buscador en inglés. Sólo se consideró la publicación original de los datos de prevalencia, de tal manera que se excluyeron publicaciones secundarias. Con excepción de un trabajo, que sólo se publicó como resumen, todos los artículos se obtuvieron in extenso.

Por carecer de las distribuciones originales de los datos publicados, fue imposible uniformar el criterio utilizado para definir anemia y, en cada caso, se tomó el punto de corte informado por los autores para las concentraciones de hemoglobina en sangre, mismo que varió entre 120 y 133 g/L para mujeres no embarazadas y entre 110 y $120 \mathrm{~g} / \mathrm{L}$ para las embarazadas. Cabe mencionar que, en cada caso, se ajustó por altitud.

Para evaluar la calidad de los estudios de intervención se buscaron los ensayos clínicos controlados informados hasta la fecha y se clasificaron de acuerdo con su nivel de evidencia, en el que se asignaron "puntos de calidad" con base en la escala validada de Jadad y colaboradores. ${ }^{14}$ Esta escala otorga dos puntos para la asignación al azar, dos para el enmascaramiento y uno más para la descripción de los abandonos y pérdidas durante el seguimiento; la puntuación máxima es de cinco puntos.

Por último, se revisaron las normas de la SSA en materia de prevención y manejo de la anemia en mujeres en edad reproductiva. De manera independiente, dos de los autores se encargaron de revisar los estudios incluidos para obtener las variables analizadas.

Para el análisis de las tendencias de la prevalencia de anemia (\%) se realizó una regresión lineal ponderada por el tamaño muestral, misma que se calculó con base en el inverso de la probabilidad de selección (STATA 8.0). Debido a que los valores que informaban eran cuestionables, se excluyeron los estudios en embarazadas de Báez-Flores y colaboradores, ${ }^{15}$ con una prevalencia de 98.2 y $99 \%$ de anemia para el segundo y 
tercer trimestres de gestación, y los de Canto de Cetina y colaboradores ${ }^{17}$ y Black y colaboradores ${ }^{16}$ en no gestantes; los autores de estos estudios encontraron una frecuencia de anemia de 74 y $54 \%$, respectivamente. En el caso de los trabajos en los que se informó la prevalencia de anemia por trimestre de embarazo, se calculó el valor promedio a fin de contar con un solo dato.

\section{Resultados}

En PubMed se identificaron 52 artículos; sin embargo, se eliminaron 33 debido no sólo a los criterios de exclusión preestablecidos, sino porque los buscadores arrojaron información sobre artículos en varones, otros grupos etáreos o en mujeres de poblaciones con ascendencia mexicana pero no residentes en el territorio nacional. En una segunda búsqueda, en la que se excluyó la palabra iron, se localizaron tres trabajos más.

Se encontró una alta correlación entre los artículos identificados en las bases de PubMed y ARTEMISA. De hecho, sólo hubo uno detallado en esta última base que no se localizó a través de PubMed. La mayoría de los artículos se identificaron en forma manual, ya que correspondieron a publicaciones previas a la era electrónica.

En total se incluyeron 46 trabajos de investigación, de los cuales 34 informaron sobre la prevalencia de anemia, ya fuera por corresponder este valor a un estudio transversal o por representar la condición basal de una intervención; nueve correspondieron a estudios clínicos -tres realizados en no embarazadas y seis en gestantes-, cuatro a programas operativos y uno a mujeres lactantes.

\section{Estudios sobre prevalencia}

En el cuadro I se describen los 34 estudios sobre prevalencia publicados entre 1939 y 2005. De éstos, 16 informaron sobre la prevalencia de anemia o la deficiencia de hierro en gestantes, 11 en no embarazadas y seis en ambos grupos, de los cuales dos correspondieron a encuestas nacionales probabilísticas.

De los 34 trabajos, $19(0.56 \%)$ se llevaron a cabo en población que asistía a servicios de salud y $12(0.35 \%)$ en población abierta; un estudio más se realizó entre estudiantes universitarias $(0.03 \%)$ y, por último, hubo otro $(0.03 \%)$ que incluyó tanto población abierta como asistente a centros de salud, realizado por Lisker y colaboradores en Yucatán. ${ }^{18}$

Los métodos utilizados para la determinación de hemoglobina fueron cianometahemoglobina en $65 \%$ de las mediciones, de las cuales casi la mitad ( $46 \%$ ) se realizaron en forma manual, $38 \%$ con equipo de campo
(HermoCue) y el restante con equipo automatizado fijo (Coulter). En los estudios realizados entre 1937 y 1957 se utilizaron otros métodos (Técnica de Evelyn, Sheaford-Sanford y hematina alcalina) y en ocho casos no se informó la técnica empleada para la determinación de hemoglobina.

En las figuras 1 y 2 se muestra que la prevalencia ponderada de anemia en mujeres en edad reproductiva ha disminuido en los últimos 65 años. En el grupo de las no embarazadas la prevalencia ha descendido de 39.6 a $15.5 \%$, en tanto que en el grupo de las embarazadas sólo ha decaído en promedio 10 puntos porcentuales, lo que significa que aún la cuarta parte de las mujeres gestantes tienen anemia. Sin embargo, si se consideran los datos de las dos encuestas nacionales de nutrición (únicas con muestras representativas), ${ }^{19,20}$ el panorama es todavía más desalentador, pues en el caso de las embarazadas la prevalencia de anemia ha registrado un aumento al pasar de 18.2 a $26.2 \%$, tal y como ha sido informado por Shamah-Levy y colaboradores. ${ }^{21}$

Al calcular la ecuación de regresión que mejor se ajustó a la tendencia de las cifras de prevalencia de anemia para las mujeres no embarazadas, se encontró una relación lineal inversa y significativa $\left(R^{2}=67.65 \%\right.$; $p<0.001)$ que permite predecir que, de seguir la tendencia actual, se requerirán cerca de 57 años para erradicar la anemia por deficiencia de hierro en estas mujeres (figura 1) y 121 años en las embarazadas $\left(R^{2}=\right.$ $7.01 \% ; p<0.001$ ) (figura 2).

En México, López-Reyes, ${ }^{22}$ en 1939, y Robinson, Payne y Calvo, en 1944, ${ }^{23}$ llevaron a cabo los trabajos iniciales sobre prevalencia de anemia. El primero corresponde a una tesis no publicada en la que se estudió una muestra aleatoria de 200 hombres y mujeres de 2 a 58 años de edad, que habitaban en Xochimilco, en el sur de la Ciudad de México; es decir, se trata de una muestra de población abierta. En este estudio, con base en la cuenta reticulocitaria, ${ }^{*}$ la prevalencia de anemia en las mujeres $>12$ años fue de $36 \%$, mientras que para los varones de la misma edad fue de $18 \%$. El estudio de Robinson y colaboradores también se llevó a cabo en población abierta de la Ciudad de México, en el barrio de Santa Julia; incluyó a 116 mujeres no embarazadas, $15 \%$ de las cuales fueron diagnosticadas como anémicas por tener una concentración de hemoglobina $<120 \mathrm{~g} / \mathrm{L}$. Cabe mencionar que también se in-

\footnotetext{
* Aceptando como anemia $>2 \%$ de reticulocitos. Evatt B, Gibas WN, Lewis SM, McArthur JR. Fundamentos del diagnóstico hematológico. México, D.F.: CDC-Funsalud, 1995.
} 


\section{Cuadro I \\ Prevalencia de anemia en mujeres no embarazadas y embarazadas documentadas en México, SEGÚN AÑO DE PUBLICACIÓN}

\begin{tabular}{|c|c|c|c|c|c|c|c|}
\hline \multirow[b]{3}{*}{ Año, autor } & \multirow{3}{*}{$\begin{array}{c}\text { Criterio } \\
\text { Anemia Hb g/L* }\end{array}$} & \multirow[b]{3}{*}{ Núm. casos } & \multirow[b]{3}{*}{ Localidad, tipo de población } & \multicolumn{4}{|c|}{ Prevalencia de anemia \% } \\
\hline & & & & \multirow{2}{*}{$\begin{array}{c}\text { No } \\
\text { Embarazadas }\end{array}$} & \multicolumn{3}{|c|}{ Trimestre de embarazo } \\
\hline & & & & & 19 & $2 \underline{0}$ & 30 \\
\hline 1939, López-Reyes²2 & $<120$ & 150 & Cd México, población abierta & 26 & - & - & - \\
\hline 1944, Robinson ${ }^{23}$ & $<120$ & 116 & Cd México, población abierta & 15 & - & - & - \\
\hline 1946, Anderson ${ }^{46}$ & $<120$ & 101 & Cd México, población abierta & - & 6.0 & 11.3 & 11.1 \\
\hline 1949, Báez ${ }^{25}$ & $<114$ & 117 & Cd México, hospital público & - & - & 9.0 & 19.8 \\
\hline 1957, Schez-Medal ${ }^{47}$ & $S / d$ & 9951 & Cd México, hospital privado & 35.0 & - & - & - \\
\hline \multirow[t]{2}{*}{ 1963, Lisker ${ }^{18}$} & $<120$ & 86 & Sudzal,Yuc, población abierta & 9.5 & - & - & - \\
\hline & & 62 & Sitilpech,Yuc, hospital público & 16.0 & - & - & - \\
\hline 1966, Báez-Flores ${ }^{15}$ & $<120$ & 600 & Saltillo, C oah, hospital público & - & - & 98.2 & 99.0 \\
\hline \multirow[t]{2}{*}{ 1966, Balam ${ }^{48}$} & $<120$ & 217 & Costa, población abierta & 33.0 & - & - & - \\
\hline & $<130$ & 77 & Altiplano, población abierta & 22.5 & - & - & - \\
\hline 1967, Díaz ${ }^{49}$ & $<120$ & 10 & Cd México, hospital público & - & 0 & 25.0 & 71.0 \\
\hline 1967, Karchmer $^{50}$ & $<120$ & 1363 & Cd México, hospital público & - & - & - & 37.6 \\
\hline 1968, Báez-Flores ${ }^{51}$ & & 68 & Cd México, población abierta & 35.0 & - & - & - \\
\hline 1969, Loría ${ }^{26}$ & $<120$ & 82 & Cd México, hospital público & - & - & - & 19.5 \\
\hline 1969, Schez-Medal ${ }^{52}$ & $<120$ & 143 & Huamantla,Tlax, hospital público & 8.3 & - & 21.3 & 27.9 \\
\hline 1971, C Ook $^{53}$ & $<120$ & 899 & Latinoamérica, hospital público & 7.3 & - & - & 38.5 \\
\hline 1972, Loría27 & $<120$ & 173 & Huamantla,Tlax, hospital público & - & - & 22.9 & 18.1 \\
\hline 1978,Vásquez ${ }^{54}$ & $<12.2$ & 425 & Cd México, universitarias & 33.4 & - & - & - \\
\hline 1979, Loría ${ }^{28}$ & $<120$ & 337 & Cd México, hospital público & - & - & 25.4 & 19.4 \\
\hline 1980, River ${ }^{55}$ & $<120$ & 500 & Durango, hospital público & 14.0 & - & - & - \\
\hline 1985, Canto de Cetina ${ }^{16}$ & $<120$ & 505 & Mérida, 3 hospitales públicos & 74.05 & - & - & - \\
\hline \multirow[t]{2}{*}{ 1994, Black ${ }^{17}$} & $<133$ & 85 & Solis, Edo Mex, población abierta & 54.0 & - & - & - \\
\hline & $<123$ & 71 & Solis, Edo Mex, población abierta & - & & $35.0^{\ddagger}$ & - \\
\hline \multirow[t]{2}{*}{ 1995, Martínez $z^{19}$} & $<120$ & 15146 & Encuesta probabilística nacional & 15.4 & - & - & - \\
\hline & $<110$ & 742 & Encuesta probabilística nacional & - & - & $18.2^{\ddagger}$ & - \\
\hline 1996, Pfeffer ${ }^{56}$ & $<125$ & 82 & Cd México, hospital público & - & 31.6 & 20 & 26.3 \\
\hline 1997, Gtz-Romero ${ }^{57}$ & $<110$ & 500 & Monterrey, N L, hospital público & - & - & 16.4 & 22.0 \\
\hline $1997, Q$ uinter0 58 & $<120$ & 77 & Morelos, población abierta & - & - & $20^{\ddagger}$ & - \\
\hline 1998, Rmz-Mateos ${ }^{59}$ & $<120$ & 490 & León, G to, hospital público & - & 8.0 & 31.0 & 49.0 \\
\hline \multirow[t]{2}{*}{ 1998, River a ${ }^{20}$} & $<120$ & 10284 & Encuesta probabilística nacional & 20.0 & - & - & - \\
\hline & $<110$ & 397 & & & & $26.2^{\ddagger}$ & - \\
\hline 2000, C asanueva $^{60}$ & $<130$ & 117 & Cd México, hospital público & 11.9 & - & - & - \\
\hline 2001,Vqz-Molina 30 & - & 163 & Clínica IMSS Chihuahua & & & & $s / d^{\S}$ \\
\hline \multirow[t]{2}{*}{ 2001, Monarrez ${ }^{61}$} & $<120$ & 446 & Tarahumara, población abierta & 16.1 & - & - & - \\
\hline & $<110$ & 35 & Tarahumara, población abierta & - & - & 25.7 & - \\
\hline 2002, Jaime-Pérez ${ }^{62}$ & $<110$ & 201 & Monterrey, hospital público & - & - & - & 46.0 \\
\hline $2003, N$ avarro ${ }^{29}$ & $\#$ & 35 & Colima, hospital público & - & 0 & 11.7 & 43.7 \\
\hline 2004, Ramakrishnan ${ }^{43}$ & $<110$ & 290 & Morelos, población abierta semirural & - & 13.4 & - & - \\
\hline 2004, Palacios ${ }^{63}$ & - & 112 & Sinaloa, población abierta rural & 54.9 & - & - & - \\
\hline 2005, 0 'Connor ${ }^{64}$ & $<133$ & 68 & Capulhuac, Edo Mex, población abierta & $61.8^{\&}$ & - & - & - \\
\hline \multirow{2}{*}{\multicolumn{8}{|c|}{$\begin{array}{l}\text { * Valores ajustados al nivel del mar } \\
\text { ₹ No se específica edad gestacional }\end{array}$}} \\
\hline & & & & & & & \\
\hline \multicolumn{8}{|c|}{$\begin{array}{l}\text { No informa prevalencia de anemia, sólo indica una hemoglobina promedio de } 120 \pm 10 \mathrm{~g} / \mathrm{L} \text { en la semana } 370 \text { más de gestación, pero señala puntos de } \\
\text { corte para ferritina }\end{array}$} \\
\hline \multicolumn{8}{|c|}{ * 1er trim <120 g/L, 2do trim <110 g/L, 3er trim $104 \mathrm{~g} / \mathrm{L}$} \\
\hline
\end{tabular}




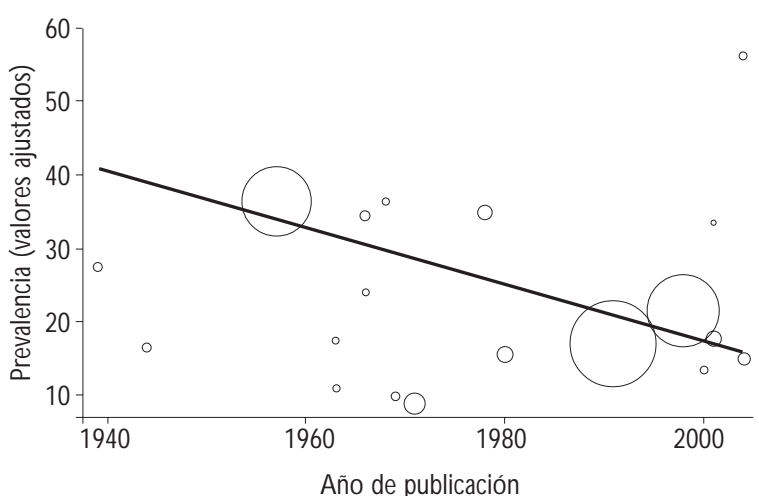

Prevalencia $=802.84-0.39 *$ Año

$p=0.001, R^{2}=62.59 \%$

Escala: 500 mujeres $=0$

Figura 1. Prevalencia de anemia en mujeres no embarazadas ponderada por tamaño muestral. México, 1940-2005

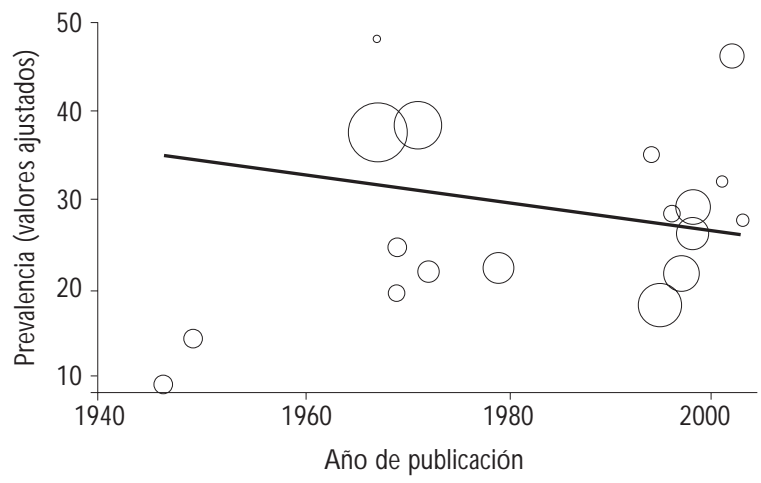

Prevalencia $=337.68-0.16 *$ Año

$p=0.001, R^{2}=7.01 \%$

Escala: 500 mujeres $=0$

Figura 2. Prevalencia de anemia en mujeres embarazadas PONDERAda por el tamaño MUestral. MéXico, 1940-2005

cluyó a 22 mujeres embarazadas o lactantes de las que se informó una prevalencia de anemia de $50 \%$. En ambas investigaciones se concluyó que la anemia por deficiencia de hierro representaba un problema de salud pública que ameritaba atención.

Es importante destacar que, en la mayoría de los estudios realizados en embarazadas, se utilizó el mis- mo punto de corte para el diagnóstico de anemia, independientemente de la etapa de gestación en que se encontrara la mujer, a pesar de existir valores de referencia para juzgar la hemoglobina con base en la edad gestacional. ${ }^{24}$ No obstante, se observaron algunas excepciones al respecto: la primera es el trabajo de BáezVillaseñor y Gómez ${ }^{25}$ quienes establecieron el volumen sanguíneo midiendo el cambio de la densidad óptica del plasma, mediante la técnica de azul de Evans. Los autores encontraron que las gestantes tenían, en promedio, $24.9 \%$ más volumen sanguíneo que las no gestantes y que a la altura de la Ciudad de México, la hemodilución conducía a una concentración de hemoglobina de $114 \mathrm{~g} / \mathrm{L}$, por lo cual propusieron el uso de esta cifra como punto de corte para considerar anemia. Adicionalmente, establecieron la validez de este criterio al contrastar los resultados del resto de los indicadores hematológicos en gestantes con cifras por arriba y por debajo del punto de corte, es decir, verificaron sus resultados a través de indicadores fisiológicos. La otra excepción son los trabajos de Loría y colaboradores, ${ }^{26-28}$ quienes sistemáticamente evaluaron sus resultados en función de la edad gestacional y propusieron diversos indicadores de acuerdo con las semanas de embarazo. Por último, Navarro-Nuñez y colaboradores ${ }^{29}$ utilizaron criterios $a d$ hoc para el diagnóstico de la anemia por trimestre.

Llama la atención que entre los estudios donde se presentan datos sobre prevalencia, sólo un grupo de investigación interrogó sobre el consumo regular de suplementos de hierro durante la gestación. ${ }^{30}$ Los investigadores encontraron que, en población derechohabiente del Instituto Mexicano del Seguro Social (IMSS), únicamente $76 \%$ de las gestantes tomó suplementos de hierro y, de éstas, $45 \%$ mostró ferritina $<11.9 \mathrm{mg} / \mathrm{L}$, mientras que $62 \%$ de las que no habían consumido suplementos presentaban reservas bajas de hierro.

Cabe destacar el hecho de que se localizó un solo estudio realizado en mujeres lactantes a pesar de que, por su condición fisiológica, también se encuentran en riesgo.

\section{Estudios de intervención}

En el cuadro II se resumen las intervenciones preventivas o terapéuticas que se han realizado en mujeres no embarazadas. El primer esfuerzo data de hace más de 230 años y se debió a José Ignacio Bartolache, cuando dio noticia sobre la fabricación de unas pastillas llamadas marciales o fierro fútil, elaboradas con base en otro medicamento fabricado por el médico Facino Gibelini en 1767, en Génova (Mercurio Volante, 


\section{Cuadro II \\ Medidas preventivas o terapéuticas en Contra de LA ANEMia en MUJeres en edAd Reproductiva no embarazadas eValuadas en México}

\begin{tabular}{|c|c|c|c|c|c|}
\hline \multirow{2}{*}{$\begin{array}{l}\text { Año, autor } \\
\text { 1774, Bartolache }{ }^{31}\end{array}$} & \multicolumn{3}{|c|}{ Tipo de programa } & \multirow{2}{*}{\begin{tabular}{l}
\multicolumn{1}{c}{ Estrategia } \\
Distribución subsidiadacon \\
instrucciones especiales \\
para población indígena
\end{tabular}} & \multirow{2}{*}{$\begin{array}{l}\text { Resultado } \\
\text { Sin evaluación }\end{array}$} \\
\hline & Preventivo & 0 perativo & $\begin{array}{l}\text { Suplementación con } \\
\text { pastillas de hierro }\end{array}$ & & \\
\hline 1999, Secretaría de Salud 32 & Preventivo & 0 perativo & $\begin{array}{l}\text { A dición de hierro y } \\
\text { otros nutrimentos }\end{array}$ & $\begin{array}{l}30 \text { mg de hierro por kg de } \\
\text { harina de maíz o de trigo } \\
\text { junto con otros nutrimen- } \\
\text { tos ( } 17.2 \% \text { de la recomen- } \\
\text { dación en cada pan y } 13.8 \% \\
\text { en cada tortilla) }\end{array}$ & Sin evaluación publicada \\
\hline 2000, Oportunidades ${ }^{38}$ & Preventivo/ terapéutico & 0 perativo & $\begin{array}{l}\text { Suplementación con } \\
\text { hierro }\end{array}$ & & Sin evaluación publicada \\
\hline $\begin{array}{l}\text { 2002,Arranque parejo } \\
\text { en la vida }{ }^{39}\end{array}$ & Preventivo/ terapéutico & 0 perativo & $\begin{array}{l}\text { Suplementación con } \\
\text { hierro como parte de } \\
\text { un multivitamínico }\end{array}$ & & Sin evaluación publicada \\
\hline 2002, Reynoso-Gómez 33 & Terapéutico & Experimental & $\begin{array}{l}\text { Hierro intravenoso } \\
(n=39)\end{array}$ & $\begin{array}{l}\text { Dosis necesaria para corre- } \\
\text { gir el déficit de hierro }\end{array}$ & $\begin{array}{l}\text { A las } 8 \text { semanas aumen- } \\
\text { to de } \mathrm{Hb} \text { de } 78 \mathrm{H} /-16.7 \\
\mathrm{~g} / \mathrm{L} \text { a } 134+/-10.8 \mathrm{~g} / \mathrm{L} \\
32 \% \text { con reacciones ad- } \\
\text { versas }\end{array}$ \\
\hline 2003, G arcía ${ }^{34}$ & Preventivo & Experimental & $\begin{array}{l}\text { A dición de vitamina C } \\
\text { a dieta habitual. Grupo } \\
\text { placebo }(n=18 \mathrm{c} / \mathrm{u})\end{array}$ & $\begin{array}{l}\text { Consumo de una limonada } \\
\text { ( } 25 \text { mg vit .C) en el desayu- } \\
\text { no y la comida por } 14 \text { días }\end{array}$ & $\begin{array}{l}\text { No mejora el estado nu- } \\
\text { tricio de hierro }\end{array}$ \\
\hline 2004, Moriarty-Craige ${ }^{36}$ & Preventivo & Experimental & $\begin{array}{l}\text { Suplementación con } \\
\text { hierro }(n=158)\end{array}$ & $\begin{array}{l}60 \text { mg hierro vs } 60 \text { mg hie- } \\
\text { rro + multivitamínico (MV) }\end{array}$ & $\begin{array}{l}\text { El Fe + MV no es más } \\
\text { efectivo que el Fe para } \\
\text { mejorar la } \mathrm{Hb}\end{array}$ \\
\hline
\end{tabular}

17 de julio de 1774). De acuerdo con el autor, la aceptación del medicamento fue muy limitada pues entre los académicos prevalecía la opinión de que el hierro podía causar daños a la salud. En un intento de poner fin a la disputa sobre la conveniencia o no del consumo de pastillas de hierro, Bartolache ${ }^{31}$ publicó un número especial del Mercurio Volante, el 19 de agosto de 1774, con las instrucciones para el "buen uso de las pastillas marciales". Ahí se señalaba que el medicamento no era un remedio universal y se aclaraban las contraindicaciones de uso. Al mismo tiempo, se exhortaba a que lo consumieran las mujeres que presentaban "abortos por debilidad, palidez con extenuación, flujos blancos y de sangre, hipocardia y mal histérico". En cuanto a la dosis, el autor indicaba que se podían tomar una, dos o tres tabletas cada dos o tres días y que su precio sería de un peso por onza para "los españoles y personas que puedan indemnizar los costos, pero que para los indios su precio sería menor". Por último, y preocupado por el buen uso que se hiciera de las pastillas, en cada receta adjuntaba un instructivo en español y otro en "idioma mexicano para los indios que no son ladinos".
En 1999 la Secretaría de Salud obliga a que las harinas de maíz y trigo se adicionen con hierro y otros nutrimentos. ${ }^{32}$ Esta medida, al igual que la propuesta por Bartolache ${ }^{31}$ no han sido evaluadas.

De los ensayos clínicos hechos en este grupo, uno señala la eficacia de la terapia intravenosa para corregir la anemia severa, pero no incluye grupo de comparación; ${ }^{33}$ otro informa que no hay mejora del estado nutricio de hierro al aumentar el consumo de vitamina $\mathrm{C}^{34}$-precedido por un estudio realizado en la misma población y con isótopos estables, en el que se informó el posible efecto benéfico del ácido ascórbico para aumentar la disponibilidad del hierro- ${ }^{35}$ y uno más que muestra un resultado positivo de la suplementación con hierro en la prevención de anemia. ${ }^{36}$ Los trabajos de García y colaboradores y de Moriarty-Craige y colaboradores, ${ }^{34,36}$ obtuvieron 3 y 5 puntos, respectivamente, de acuerdo con la escala de Jadad.

En lo que se refiere a la evaluación de intervenciones en gestantes (cuadro III), en 1993 la Secretaría de Salud publicó en el Diario Oficial de la Federación una norma en la que se establece la obligatoriedad de su- 


\section{Evaluación de intervenciones para preveniR y combatir la anemia en gestantes en México}

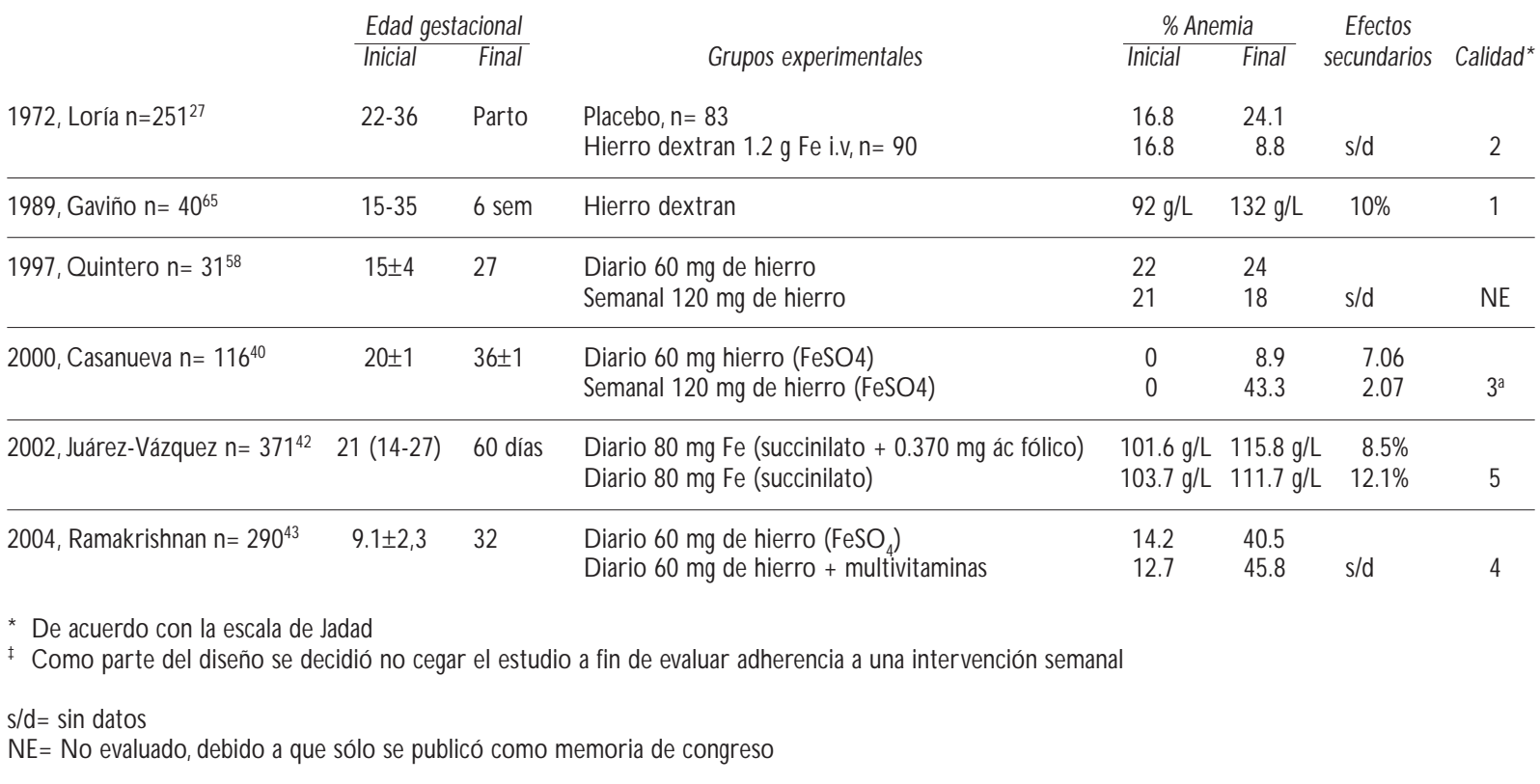

plementar con hierro y ácido fólico a toda mujer gestante, aunque no se indican ni dosis ni frecuencia de la misma. ${ }^{37}$ Posteriormente, en 1997 y 2002 se establecieron los programas "Oportunidades" (antes "Progre$\left.\mathrm{sa}^{\prime \prime}\right)^{38}$ y Arranque parejo en la vida, ${ }^{39}$ respectivamente, que se han enfocado a los grupos en pobreza extrema, especialmente a las mujeres y los niños. El primero dota a todas las embarazadas con una bebida de alta densidad energética, Nutrivida, que contiene $15 \mathrm{mg}$ de hierro, en tanto que el segundo promueve el consumo de multivitamínicos en las mujeres adultas, sin informar la dosis. Aún no se ha publicado la evaluación de ambas intervenciones.

En este grupo sólo existen seis estudios clínicos que exploran la suplementación con hierro, y de ellos uno que evaluó estrictamente el efecto preventivo ${ }^{40}$ (cuadro III). El trabajo de Loría y colaboradores fue el pionero y tuvo, además, la ventaja de que evaluó el efecto de la dosificación con hierro tanto en la madre como en el recién nacido e, inclusive, valoró el efecto de la suplementación materna sobre el crecimiento del niño ${ }^{41}$ Tres de los ensayos clínicos ${ }^{40,42,43}$ obtuvieron tres puntos o más en la evaluación de calidad; uno de ellos fue multicéntrico. ${ }^{42}$

\section{Discusión}

Antes de abordar esta sección es necesario señalar algunas de las limitaciones metodológicas del estudio. Por tratarse de un meta-análisis de estudios epidemiológicos observacionales se carece de los datos originales, por lo que no fue posible controlar algunos posibles confusores como el punto de corte de hemoglobina utilizado para diagnosticar anemia o el nivel socioeconómico. Los avances tecnológicos se reflejan en el cambio de técnicas empleadas para la medición de hemoglobina; empero, se debe destacar que $65 \%$ de los estudios refieren haber utilizado la técnica de cianometahemoglobina, lo que brinda consistencia a la información. Más aún, se ha informado una alta correlación entre los resultados automatizados realizados con equipo fijo y de campo $(>97 \%){ }_{1}^{44}$ por lo que la tendencia secular de la anemia probablemente no es atribuible a la técnica de medición.

La revisión también refleja los cambios que se han producido en el estudio de distintas enfermedades, es decir, en la mayoría de los casos se inicia con trabajos en los que se describe el cuadro clínico, para después estudiar poblaciones con alto riesgo de presentar la alteración o enfermedad y, por último, se inician los estudios en muestras probabilísticas. En el caso que nos ocupa, los trabajos realizados entre 1940 y 1995 corresponden a las dos primeras etapas (estudios clínicos y encuestas en grupos de población marginada o asistentes a servicios de salud) y únicamente a partir de esa fecha se han realizado estudios en muestras representativas de la población.

En México, como en el resto del mundo, la anemia por deficiencia de hierro es una de las carencias más 
estudiadas, ${ }^{45}$ a pesar de ello, las medidas tomadas para su prevención y manejo todavía son muy limitadas, particularmente en lo que se refiere a las mujeres en edad reproductiva. Esto se debe, fundamentalmente, a que la gran mayoría de las investigaciones se han centrado en explorar su prevalencia ( $78 \%$ de los trabajos publicados) y sólo en fechas recientes se han empezado a realizar estudios clínicos para evaluar la eficacia y efectividad de las intervenciones (cuadros II y III). Dentro de los estudios sobre prevalencia, cabe destacar que los puntos de corte utilizados para diagnosticar anemia no son uniformes ni reconocen los cambios fisiológicos que supone la gestación, es decir, utilizan el mismo valor independientemente de la etapa del embarazo, por lo que las prevalencias informadas no son necesariamente confiables.

Llama la atención que, a pesar de la magnitud del problema, sólo fue posible identificar seis estudios de intervención. Los más evaluados están relacionados con la suplementación terapéutica; Loría y coaboradores publicaron el primero (utilizando hierro dextran en dosis única) ${ }^{27}$ y en años más recientes han aparecido cuatro estudios en los que se evalúa la eficacia de hierro como suplemento durante el embarazo, ya sea sólo o acompañado con otras vitaminas, en esquemas diarios o semanales. Sin embargo, los resultados no son consistentes.

Respecto a las intervenciones con resultados de mediano plazo, tampoco se cuenta con evaluaciones de las bondades de la suplementación obligatoria con hierro durante la gestación, e incluso en las encuestas nacionales de nutrición realizadas es imposible evaluar el apego a esta norma, dado que no se exploró. Una excepción es el trabajo realizado por VásquezMolina y colaboradores ${ }^{30}$ en el IMSS de Chihuahua en el que, además, se informa sobre la estrategia de pinzamiento del cordón umbilical.

Por otra parte, existen aún menos estudios de intervención en mujeres no embarazadas; el único que evalúa la suplementación en mujeres no gestantes se realizó en una población rural y mostró que administrar hierro diariamente es igual de efectivo para prevenir la anemia que el uso de este nutrimento como parte de un multivitamínico. ${ }^{36}$ Cabe señalar que no hay trabajos que analicen la efectividad de la suplementación semanal como medida preventiva.

Una intervención de largo plazo dirigida a la población general es la adición de hierro a las harinas, pero ésta no necesariamente forma parte de una política de adición de alimentos y todavía no ha sido evaluada.

Así, ante la carencia de información suficiente y sistemática, no resulta extraño que en México la anemia en mujeres en edad reproductiva, y particular- mente en las embarazadas, siga siendo un problema de salud pública. Si bien es cierto que la prevalencia de anemia ha disminuido a partir de la segunda mitad del siglo pasado, todavía afecta a $15 \%$ de las mujeres no gestantes y a $25 \%$ de las embarazadas. Lo anterior significa que, siguiendo los esquemas actuales, serán necesarios más de 50 años para erradicar la anemia entre las no gestantes y más de 120 en las embarazadas. Más aún, si sólo se consideran los resultados de las únicas encuestas probabilísticas, la prevalencia de anemia tanto en mujeres no gestantes como en embarazadas muestra un incremento en un periodo relativamente corto. No obstante, es importante tomar en cuenta que en fechas recientes se han puesto en marcha una serie de medidas dirigidas a grupos de alta vulnerabilidad para la deficiencia de hierro como son los programas Oportunidades, Arranque Parejo y Salud y nutrición de la población indígena, lo que probablemente permitirá revertir la tendencia en el mediano plazo.

En conclusión, puede afirmarse que, a pesar de estar bien documentada la elevada prevalencia de anemia por deficiencia de hierro entre las mujeres en edad reproductiva, no se han logrado avances significativos en su combate debido a que las acciones emprendidas son pequeñas, no están sistematizadas o se carece de mecanismos efectivos de evaluación.

Es necesario obtener más evidencia referente a las dosis y a los esquemas de suplementación con hierro que involucre poblaciones con diferentes estados de nutrición y de salud-enfermedad en distintos escenarios sociales y que permita establecer, a partir de la misma, las bases de las intervenciones orientadas a erradicar la anemia de una forma más acelerada. Además -y a pesar de no ser un punto estrictamente relacionado con el estudio de la prevalencia de anemia-, es importante retomar parte de la experiencia y, al igual que en los primeros trabajos del siglo anterior, reflejar un interés genuino por las condiciones y problemas de las mujeres estudiadas, más allá de las cifras.

\section{Referencias}

1.Allen LH. Multiple micronutrients in pregnancy and lactation:An overview. Am J Clin N utr 2005;81 suppl:1206s-1212s.

2.Allen LH.Anemia and iron deficiency: effects on pregnancy outcome. A m J Clin N utr 2000; 71 suppl 5: 1280s-1284s.

3. Hercberg S, Galan P, Preziosi P,Aissa M. Consequences of iron deficiency in pregnant women. Clin Drug Invest 2000; 19 suppl 1: 1-7. 4. Rush D. N utrition and maternal mortality in the developing world.Am J Clin N utr 2000; 72 Suppl 1:212s-240s . Erratum in 2001; 73:134

5. Kaufer M, Casanueva E. Relation of prepregnancy serum ferritin levels to hemoglobin levels throughout pregnancy. Europ J Clin N utr 1990; 44:709-15. 
6. C asanueva E, Pfeffer F, D rijanski A, Fernández-G axiola AC, GutiérrezValenzuelaV, Rothenberg SJ. Iron and folate status before pregnancy and anemia during pregnancy. Ann N utr Metab 2003; 47:60-63.

7. Brownlie T 4th, Utermohlen V, Hinton PS, Haas JD.T issue iron deficiency without anemia impairs adaptation in endurance capacity after aerobic training in previously untrained women. Am J C lin N utr 2004;79:437-443

8. Kim SH, Kim HY, Kim W K, Park OJ. N utritional status, irondeficiency-related indices, and immunity of female athletes. N utrition 2002;18:86-90.

9. Perez EM, Hendricks MK, Beard JL, Murray-Kolb LE, BergA,Tomlinson $M$, et al. Mother-infant interactions and infant development are altered by maternal iron deficiency anemia. J N utr 2005;135:850-855. 10.Viteri FE. Prevention of iron deficiency. En: Prevention of micronutrients deficiencies. Tools for policymakers and public health workers.W ashington: $N$ ational A cademy Press, 1998: 45-102.

11. Freire W B. Anemia por deficiencia de hierro. Estrategias para su prevención O PS/O MS. Salud Publica Mex 1998;40(2):199-205.

12.IN ACG -W HO -UN ICEF guidelines for the use of supplements to prevent and treat iron deficiency anemia. Stoltzfus RJ, D reyfus $M L$, ed. W ashington DC: ILSI Press,1998.

13. Stroup DF, Berlin JA, Morton SC, O lkin I,W illiamson GD, Rennie D et al. Meta-analysis of observational studies in epidemiology:A proposal for reporting. Meta-analysis of O bservational Studies in Epidemiology (MO O SE) group. JAMA 2000;283:2008-2012.

14.Jadad AR, Moore RA, Carroll D, Jenkinson C, Reynolds DJ, Gavaghan $D$ J, et al. Assessing the quality of reports of randomized clinical trials: is blinding necessary? Control C lin Trials 1996;17:1-12.

15. Báez-Flores M, C hávez-Villasana A, Trimer- Hernández C, Lara JM. La anemia en el embarazo. Estudio de 600 embarazadas de la ciudad de Saltillo, Coah. Salud Publica Mex 1966; 8: 573-579.

16. C anto de Cetina TE, Cardenas S, 0 rtiz ME, Polanco L,Vera L, Pina $C$ astro $R$, et al. Valores de hemoglobina y hierro sérico en mujeres de clase socioeconómica baja,Yucatán, México. Bol 0 ficina Sanit Panam $1985 ; 98(5): 464-472$.

17. Black AK, Allen L, Pelto G, Mata M, C havez A. Iron, vitamin B12 and folate status in Mexico:Associated factors in men and women and during pregnancy and lactation.J N utr 1994;124:1179-1188.

18. Lisker R, Loría A. Frecuencia y características de la anemia en el medio rural mexicano. Rev Invest Clin 1963;15:29-42.

19. Martínez H, G onzález-C ossío T, Flores M, Rivera-D omarco J, Lezana MA, Sepúlveda-A mor J.Anemia en mujeres de edad reproductiva. Resultados de una encuesta probabilística nacional. Salud Publica Mex 1995; 37:108-119.

20. Rivera-D omarco J, Shama-Levy T,Villalpando-Hernández S, G onzález de Cossío T, Hernández-Prado B, Sepúlveda J. Encuesta N acional de Nutrición 1999. Estado nutricio de niños y mujeres de México. Cuernavaca, Morelos, México: Instituto N acional de Salud Pública, 2001. 21. Shamah-Levy T,Villalpando S, Rivera JA, Mejia-Rodriguez F, C amachoCisneros M, Monterrubio EA. Anemia en mujeres mexicanas. Un problema de salud pública. Salud Publica Mex. 2003;45 suppl 4:S499-507. 22.López-Reyes EM. Los reticulocitos en la sangre humana (tesis). México, D F: Facultad de Ciencias Q uímicas y Físicas, U niversidad N acional Autónoma de México, 1939.

23. Robinson W D, Payne GC, Calvo J.A study of the nutritional status of a population group in Mexico City. J Am Diet Assoc 1944;20:289-97. 24. CDC (USA). Criteria for anemia in children and childbearing-aged women. MMW R 1989;38: 400-404.

25. Báez-Villaseñor, Gómez M. Estudios hematológicos en el embarazo: cuadro hemático, volumen sanguíneo y tendencia hemorrágica. Rev Invest Clin 1949:1:279-303.
26. LoríaA, Sánchez-Medal L, Labardini J, Soberón J, Karchmer S. Comparación entre el estado nutricional de la madre y del recién nacido. Gac Med Mex 1969; 99:229-239.

27. Loría A, C ordourier E,Arroyo P, Piedras J, Sánchez-Medal L. Anemia nutricional IV. Hierro dextran en dosis intravenosa única en la profilaxis de la anemia hipoferrémica del embarazo. Rev Invest Clin 1972; 24: 113-122.

28. Loría A, A rroyo P, Piedras J, Sánchez-Medal L.Anemia en el embarazo II. Datos hematológicos y obstétricos en embarazadas de dos instituciones mexicanas. Rev Invest Clin 1979;31:217-230.

29. N avarro-N uñez C , D el Toro-Equihua C, A guayo-G odínez A, Venegas0 choa U, Muñiz-Gómez A. A nemia en embarazadas residentes en área rural a 540 metros de altitud. Ginecol 0 bstet Mex 2003;71:124-130. 30.Vásquez-Molina ME, Corral-Terrazas M, A pezteguia MA, C armonaSawasky J, Levario-C arrillo M. Relación entre las reservas de hierro maternas y del neonato. Salud Publica Mex 2001;43:402- 407.

31. Moreno R. José Ignacio Bartolache, Mercurio Volante (1772-1773). México: Universidad N acional Autónoma de México, 1993: 177-183. 32.Rosado JL, Camacho-Solis R, Bourges H. Adición de vitaminas y minerales a harinas de maíz y trigo en México. Salud Publica Mex 1999; 41:130-137.

33. Reynoso-Gomez E, Salinas-Rojas V, Lazo-Langner A. Safety and efficacy of total dose intravenous iron infusion in the treatment of irondeficiency anemia in adult non-pregnant patients. Rev Invest $\mathrm{Clin}$ 2002;54(1):12-20

34. García O P, Díaz M, Rosado JL,Allen LH.Ascorbic acid from lime juice does not improve the iron status of iron-deficient women in rural Mexico. Am J C lin N utr 2003;78:267-273.

35. Diaz M, Rosado JL,Allen LH,Abrams S, Garcia O P.The efficacy of a local ascorbic acid-rich food in improving iron absorption from Mexican diets:A field study using stable isotopes. Am J C lin N utr. 2003;78:436-440. 36. Moriarty-Craige SE, Ramakrishnan U, N eufeld L, Rivera J, Martorell R. Multivitamin-mineral supplementation is not as efficacious as is iron supplementation in improving hemoglobin concentration in non pregnant anemia women living in Mexico. Am J C lin N utr 2004; 80: 1308-1311.

37. N O M 007 SSA2. N orma 0 ficial Mexicana para la atención de la mujer durante el embarazo, parto y puerperio y del recién nacido, Secretaría de Salud 1993.

38. Rivera JA, Rodríguez G, Shamah T, Rosado JL, Casanueva E, Maulén I, et al. Implementation, monitoring, and evaluation of the nutrition component of the Mexican Social Program (PRO GRESA). Food N utr Bull 2000; 21:35-42.

39. Secretaría de Salud. Subdirección de Prevención y Protección a la Salud. Programa de Acción "Arranque parejo en la vida". 2002, México. http://www.e-local.gob.mx/wb2/ELOCAL ELOC_Programa_de_la_SSA_ Arranque_parejo_en_la_vida

40. C asanueva E,Viteri FE, Loría A, Mares-Galindo M, Meza C,ValdésRamos R. D aily of weekly iron containing supplementation in nonanemic pregnant women in Mexico City. Exp Biol 2000,Abst 359.3 41. Loría A, Sánchez-Medal L,Arroyo P, C ourdourier E, Piedras ], C asanueva E. N utritional anemiaVIII. Hemoglobin and plasma iron in infants treated prenatally with iron. N utr Rep Int 1979;19:451-463. 42. Juárez-Vázquez J, Bonizzoni E, Scotti A. Iron plus folate is more effective than iron alone in the treatment of iron deficiency anaemia in pregnancy:A randomised double blind clinical trial. BJO G 2002; 109: 1009-1014.

43. Ramakrishnan U, N eufeld L, Gonzalez-C ossío T,Villalpando S, GarcíaGuerraA, Rivera J, et al. Multiple micronutrient supplements during pregnancy do not reduce anemia or improve iron status compared to iron-only supplements in semirural Mexico. J N utr 2004;134:898-903. 
44. N eufeld L, G arcía-G uerra A, Sánchez-Francia D, N ewton-Sánchez 0 , Ramírez-Villalobos MD, Rivera-D ommarco J. Hemoglobin measured by Hemocue and a reference method in venous and capillary blood:A validation study. Salud Publica Mex 2002; 44:219-227

45. Rosado JL, Bourges H, Saint-Martin B. D eficiencia de vitaminas y minerales en México. Una revisión crítica. II minerales. Salud Publica Mex. 1995;37:130-139

46.Anderson RK, Robinson W D, C alvo J, Payne GC. N utritional status during pregnancy and after delivery of a group of women in Mexico City. J Am D iet Assoc 1946;22:588-593.

47. Sánchez Medal L, Rosillo J,A rellano T, Marquina J. Frecuencia de anemia en la Ciudad de México. Rev Invest Clin 1957;9:127-146.

48. Balam G, Chávez A. Frecuencia de anemia en algunas comunidades rurales del altiplano y de las costas. Salud Publica Mex 1966;8:225-33. 49. Díaz de Mathmann C, García S. N iveles séricos de hierro y hábitos alimentarios durante el embarazo. Rev Invest Clin 1967;19:333-342.

50. Karchmer S,A guilar GJA, G uerra Z, Santos G, C astelazo-Ayala L. $\mathrm{N}$ utrición y estado gravídico puerperal I. Correlación de los niveles de hemoglobina en la madre con la frecuencia y sobrevivencia del producto prematuro. Ginecol 0 bst Mex 1967;22:433-435.

51. Báez-Flores $M$, et al. La anemia en embarazadas de diferente nivel socioeconómico.Arch Ven N utr 1968;32:21, citado por Royston E.The prevalence of nutritional anaemia in women in developing countries: $A$ critical review of available information. World Health Stat Q 1982;35:

52-91.

52. Sánchez-Medal L, Labardini J, Pérez MA, Loría A.A nemia en el embarazo I. Estudio de 143 embarazadas de Huamantla, Tlaxcala. Gac Med Mex 1967;97:1333-1346.

53. Cook JD, Alvarado A, G utmisky M, Isamra J, Lsabardini M, Lsyrisee M, et al. $\mathrm{N}$ utritional deficiency and anemia in Latin America:A collaborative study. Blood 1971;38:591-603.

54.Vázquez-Santaella J, Benítez H, Azuara-Martínez C, de la Rosa-Lazos 0 , Killer S. Frecuencia de anemia en 1545 estudiantes de primer ingreso de la UN AM. Salud Publica Mex 1978;20:485-91.

55. Rivera-D amm R, Ruiz MR, N acarro-C havarría C, 0 rtiz-C isneros E, Almonte-Huerta $\mathrm{H}$. La prevalencia de anemia en mujeres asistentes a servicios de planificación familiar en la Ciudad de D urango. Rev Invest Clin 1980;32:369-374.

56. Pfeffer F,Valdés-Ramos R, Ávila-Rosas H, Meza C, C asanueva E. Iron, zinc and vitamin $C$ nutritional status is not related to weight gain in pregnant women. N utr Res 1996; 16:555-564.

57. Gutiérrez-Romero M, O rtiz-Bonilla B, Collazo-Jaloma J, FierroMaganda ML,A mancio-C hassin O, C arillo-Galindo A. Prevalencia de anemia en mujeres con embarazo normal de una población urbana. Rev Med Hosp Gen 1997; 60:20-25.

58. Q uintero-Gutiérrez A, Rivera-D omarco JA, González-Rosendo G. Suplementación con hierro en dosis semanal y dosis diaria en la prevención de anemia ferropriva en mujeres embarazadas. XI Congreso Latinoamericano de N utrición, Guatemala 1997:CM-088

59. Ramírez-Mateos C, Loría A, N ieto-Gómez M, Malacara JM, Piedras J. Anemia y deficiencia de hierro en 490 embarazadas mexicanas. Rev Invest Clin 1998;50:119-126.

60. C asanueva E, D rijanski A, Fernández-G axiola AC, Meza C, Pfeffer F. Folate deficiency is associated with obesity and anemia in Mexican urban women. N utr Res 2000;20:1389-1394.

61. Monarrez-Espino J, Martínez H, G reiner T. Iron deficiency anemia in Tarahumara women of reproductive-age in N orthern Mexico. Salud Publica Mex 2001;43: 392-402.

62. Jaime-Pérez JC, Gómez-A Imaguer D. Iron stores in low income pregnant Mexican women at term. Arch Med Res 2002;33: 81-84. 63. Palacios-N ava ME, Moreno-Tetlacuilo LM. D iferencias en la salud de jornaleras y jornaleros agrícolas migrantes en Sinaloa, México. Salud Publica Mex 2004; 46(4):286-293.

64. 0 'C onnor D L, Latulippe ME, C ampos C, Merlos C, Villalpando S, Picciano MF. Folate deficiency does not alter the usefulness of the serum transferrin receptor concentration as an index for the detection of iron deficiency in Mexican women during early lactation.J N utr 2005;135:144-149.

65. G aviño-A mbriz S, G avino-G avino F, C enteno-C ardenas J,A huedA hued JR. Manejo alternativo de la anemia ferropénica en el estado grávido-puerperal. Ginecol 0 bstet Mex 1989;57:247-251. 